\title{
TH3F-52
}

\section{PROPAGATION OF GAUSSIAN PULSES IN LOSSY COPLANAR WAVEGITIDES}

\author{
Chun-Lin Liao, Yu-Ming Tu, Jeng-Yi Ke, and Chun Hsiung Chen \\ Department of Electrical Engineering, \\ National Taiwan University, \\ Taipei, Taiwan 10617, ROC
}

\begin{abstract}
By comparing the results by the modified spectral-domain approach with those by the previous empirical formulas, a new attenuation constant empirical formula is proposed in discussing the conductor and leaky-wave losses associated with a practical coplanar waveguide (CPW) in which the thickness and conductivity of signal strip and ground planes are finite. Based on this new empirical formula, the transient propagation characteristics of Gaussian pulses along a lossy coplanar raveguide are investigated in detail. In particular, the transient propagated pulses calculated by different empirical formulas are discussed and compared with those of the experimental data.
\end{abstract}

\section{INTRODUCTION}

Modern development of high speed devices and circuits necessitates a more acurate timedomain analysis of transmission lines. Specifically, transient analysis of coplanar transmission line structures, such as suspended coplanar waveguides [1], slot lines and open coplanar waveguides [2]-[3], has been reported. Meanwhile, two approximate formulas were proposed for modeling the modal dispersion and attenuation of coplanar transmission lines. However, all transient propagation studies were usually conducted under the assumptions of perfectly electric conductors and zero metallization thickness [1]-[3]. In practical MMIC structures, the effect of finite thickness and finite conductivity of the metal can not be neglected [4] and a more accurate model is needed.

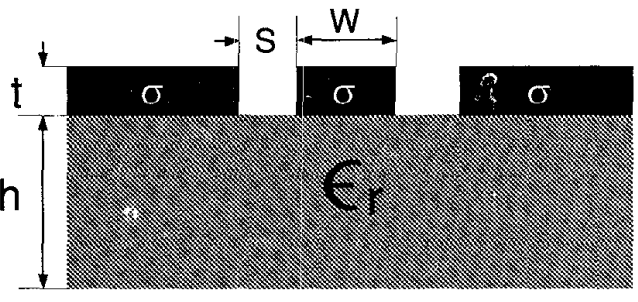

Fig. 1: Cross section of coplanar waveguide.

In this study, a new empirical formula for attenuation constant of CPW in describing conductor losses and surface-wave leakage is proposed, from which an efficient time-domain propagation model may be established.

\section{FORMULATION}

The transient propagation behavior of a Gaussian pulse along a practial coplanar waveguide as shown in Fig. 1 is the main concern of this study. To this end, the propagation constant of the waveguide $(\gamma=\alpha+j \beta)$, which includes the attenuation constant $\alpha$ and phase constant $\beta$, is needed in characterizing the time-domain wave behaviors. The propagation constant can be computed, numerically, by the time-comsuming full-wave techniques such as the modified spectral-domain approach [4]. For practical design purpose, it is better described by the empirical formulas.

One simple empirical formula [2] was proposed 
for the effective dielectric constant of an idealized coplanar waveguide. This formula was obtained under the assumption of infinitely thin conductors and infinity conductivity.

Accurate values of attenuation constant $\alpha^{S D A}$ were available by the modified spectral-domain approach [4]. But this method is too time consuming to become a practical tool for circuit design purpose. Physically, high-frequency attenuation constant of CPW is mainly due to the surfacewave leakage, and it can be approximately represented by the radiation empirical term $\alpha^{\text {rad }}$ [3]. This radiation term was derived by the assumption of infinitely thick substrate as well as zero metallization thickness and infinity conductivity. In the lower frequency region, the attenuation constant is mainly associated with the conductor loss which may be approximated discussed by a simple analytical formula $\alpha^{\text {cond }}[5]$.

However, the sum of $\alpha^{\text {rad }}$ and $\alpha^{\text {cond }}$ is inadequate in representing the more accurate $\alpha^{S D .1}$. In this study, a new corrected term $\alpha^{c}$ is proposed so that it may better fit the curves for $\left(\alpha^{S D A}\right.$ $\left.\alpha^{r a d}\right)$. After comparing the results for $\alpha^{S D A}$ and $\alpha^{\text {rad }}$, a new corrected empirical formula for attenuation constant $\alpha^{C E F}$ is established for the pratical CPW shown in Fig.1,

$$
\begin{gathered}
\alpha^{C E F}=\alpha^{r a d}+\alpha^{c} \quad(N p / m m) \\
\alpha^{c}=\frac{f(k)}{(w+2 s)} \cdot\left(\frac{f}{f_{t e}}\right)^{B} \cdot \epsilon_{r}^{0.3} \cdot g(t) \cdot\left(\frac{\sigma}{\sigma_{r}}\right)^{-0.77}
\end{gathered}
$$

where $f_{t e}=c /\left(4 h \sqrt{\epsilon_{r}-1}\right)$ is the cutoff frequency of the $T E_{1}$ mode [2], $f(k)=8.67 k^{2}-7.82 k+4.17$, $B=\left(0.25\left(\frac{t}{t_{r}}\right)+0.14\right) \cdot(0.59 k+0.73), g(t)=$ $0.01\left(\frac{t_{r}}{t}\right)^{2}+0.42\left(\frac{t_{r}}{t}\right)+0.63$, and $k=\frac{w}{(w+2 s)}$. In (1), $\omega$ and $s$ are in $\mu \mathrm{m}, \sigma_{r}$ is $1.0 \times 10^{7} \mathrm{~S} / \mathrm{m}$, and $t_{r}$ is $1.0 \mu \mathrm{m}$.

\section{NUMERICAL RESULTS}

Fig. 2 compares the effective dielectric constants $\epsilon_{e f f}$ and attenuation constants $\alpha$ of a practical CPW, based on different approaches. With the presence of the internal inductance of the conductor, the effective dielectric constant $\epsilon_{e f f}^{S D .4}$ by the modified spectral-domain approach [4] is somewhat different from that $\epsilon_{e f f}^{E F}$ by the empirical formula

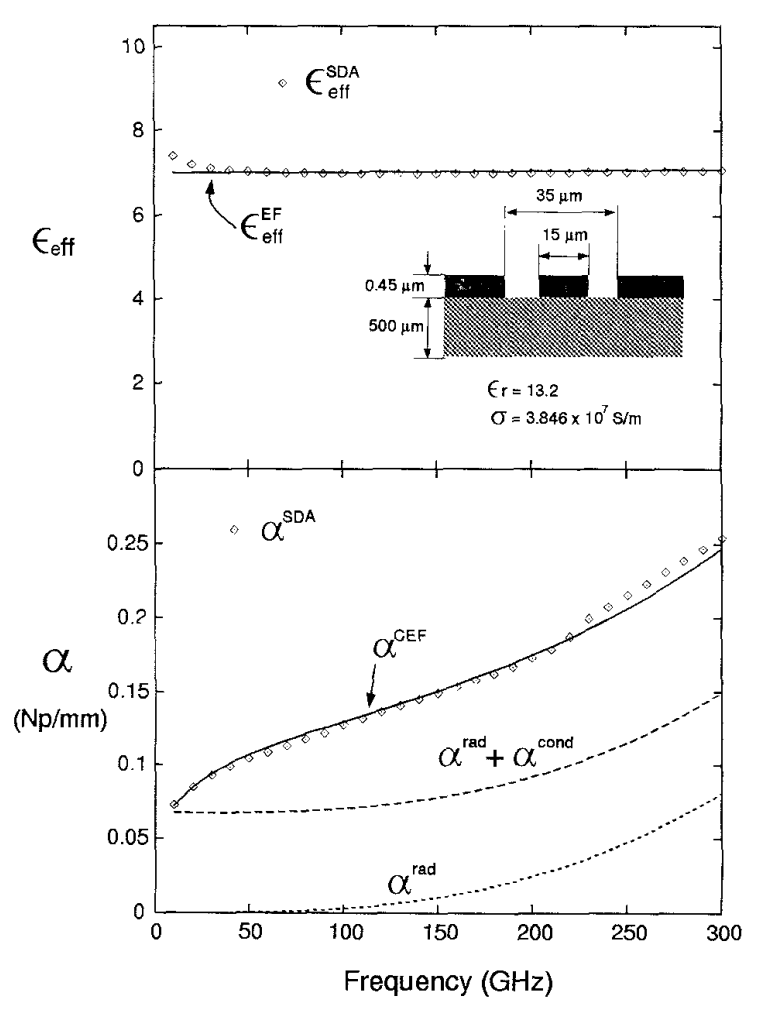

Fig. 2: Effective dielectric constant $\epsilon_{e f f}$ and attenuation constant $\alpha$ by different approaches.

[2]. But this difference is not essential in affecting the transient behavior and will be neglected in this study. The attenuation constant $\alpha^{C E F}$ by corrected empirical formula (1) is compared with that $\alpha^{S D A}$ by the modified spectral-domain approach [4]. Also included are the approximate one $\alpha^{\text {rad }}$ and the sum $\left(\alpha^{\text {rad }}+\alpha^{\text {cond }}\right)$. Good fitting between $\alpha^{C E F}$ and $\alpha^{S D A}$ curves suggests the use of the corrected empirical formula (1) in the subsequent analysis.

Shown in Fig. 3 are the simulated propagating pulses at positions $z=0,3 \mathrm{~mm}$, and $5 \mathrm{~mm}$ by three different empirical formulas. Also included are the corresponding measured pulses by [6] for comparison. Here the input is a Gaussian pulse whose full width at half maximum $(\tau)$ is $0.52 \mathrm{ps}$. The corrected empirical formula $\alpha^{C E F}$ in (1) is better than the others, $\alpha^{\text {rad }}$ and $\left(\alpha^{r a d}+\alpha^{\text {cond }}\right)$, as reflected by the good agreement with the experimental data. 

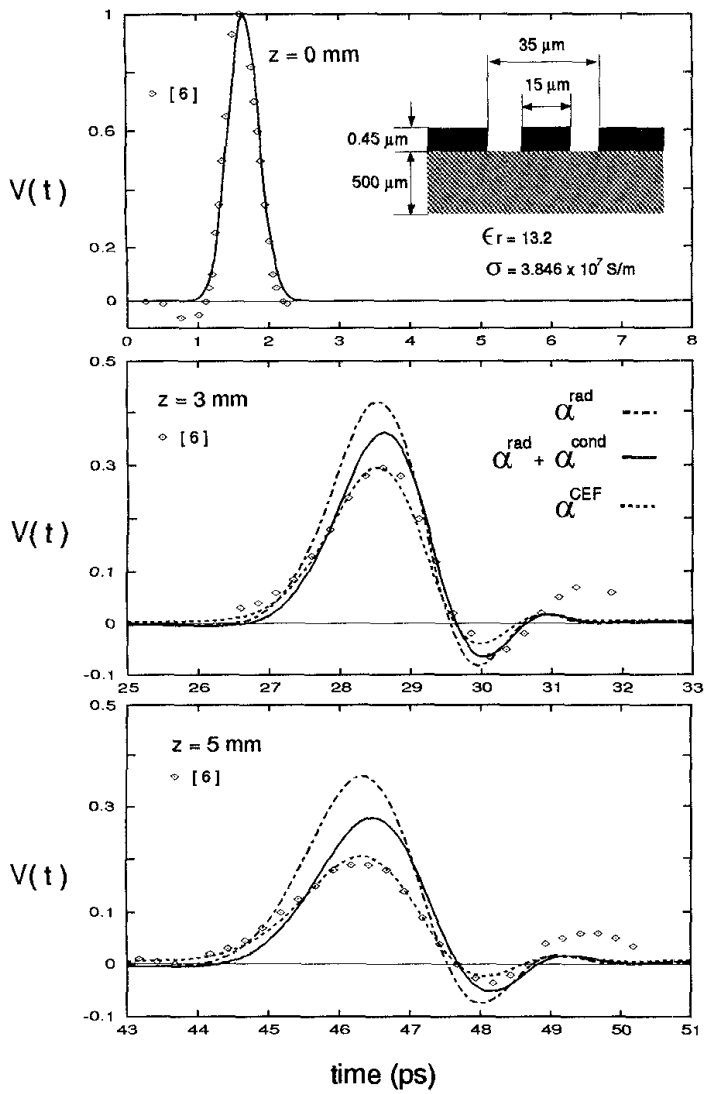

Fig. 3: Propagation of Gaussian pulses based on different empirical formulas.

Fig. 4 shows the transient waveforms with propagation distance as paremeters. Note that the tails of narrow pulses show ripples while the wide pulses do not. Physically, a broader frequency spectrum is associated with the narrow pulse whose lower frequency component has a phase velocity faster than the higher frequency component. And the appearance of ripples in pulse tails is a consequence of this difference in phase velocities.

Fig. 5 shows the waveforms for different input pulse widths at $z=3 \mathrm{~mm}, 5 \mathrm{~mm}$. The attenuation constant of CPW increases rapidly as the frequency increases, especially when the leaky surface wave is excited. The high frequency component of narrow pulse, which has broader frequency band, would suffer greater attenuation than that of wide pulse. Thus, the amplitude of narrow pulse would be lower than that of wide pulse, when they are propagated along the CPW.

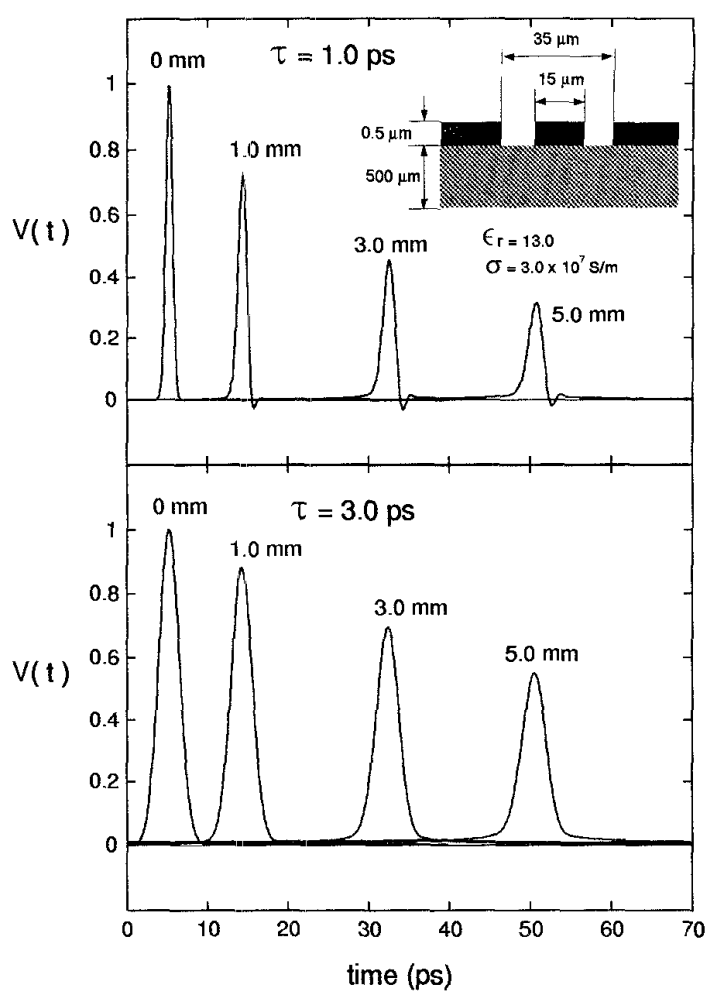

Fig. 4: Propagation of Gaussian pulses with propagation distance as parameters.

Shown in Fig. 6 are the results for characterizing the pulse shape such as peak amplitude, rise time, pulse width, and delay time. The delay times are almost the same for the three pulses, but the dispersion and attenuation become serious as the pulse becomes narrower.

\section{CONCLUSIONS}

In this work, an accurate empirical formula for discussing the conductor and leakage losses of practical coplanar waveguides with finite metallization thickness and finite conductivity has been proposed. Based on this new empirical formula, extensive propagation studies of Gaussian pulses along lossy coplanar waveguides have been conducted, and the dispersion and attenuation characteristics of lossy coplanar waveguides have been discussed. 

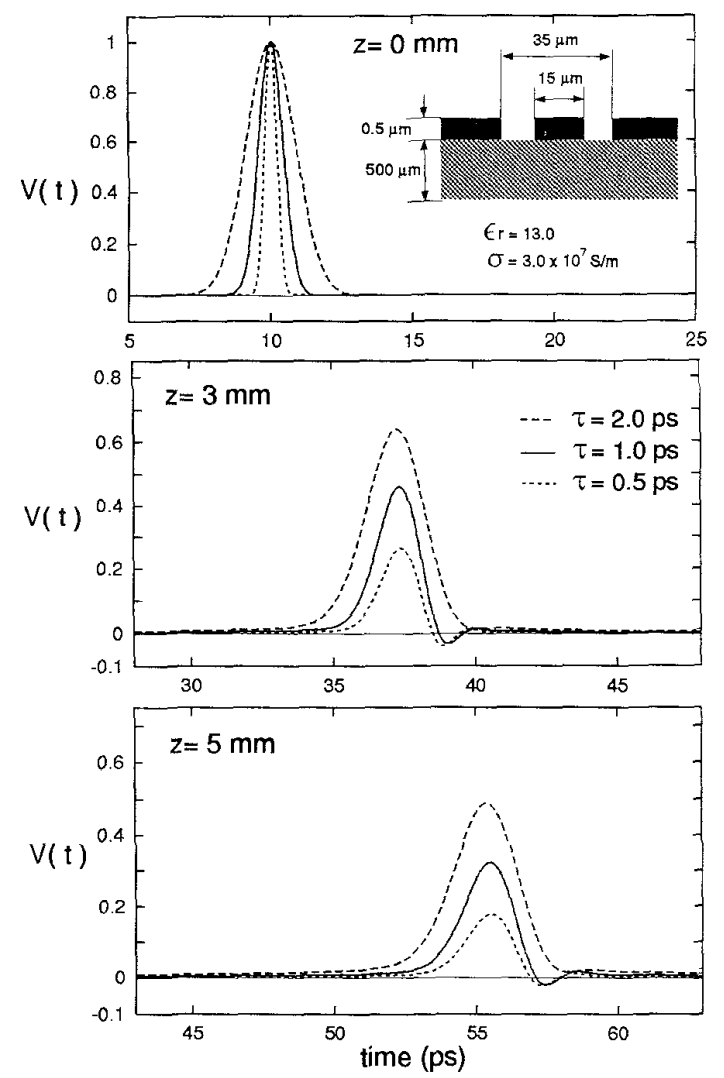

Fig. 5: Propagation of Gaussian pulses with input pulse width $\tau$ as parameters.

\section{References}

[1] Y. Qian, E. Yamashita, and K. Atsuki, "Modal dispersion control and distorsion suppression of picosecond pulses in suspended coplanar waveguides ," IEEE Trans. Microwave Theory Tech., vol. 40, pp. 1903-1909, Oct. 1992.

[2] G. Hasnain, A. Dienes, and J. R. Whinnery, "Dispersion of picosecond pulses in coplanar transmission lines," IEEE Trans. Microwave Theory Tech., vol. 34, pp. 738-741, June 1986.

[3] M. Y. Frankel, S. Gupta, J. A. Valdmanis, and G. A. Mourou, "Terahertz attenuation and dispersion characteristics of coplanar transmission lines," IEEE Trans. Microwave Theory Tech., vol. 39, pp. 910-916, June 1991.

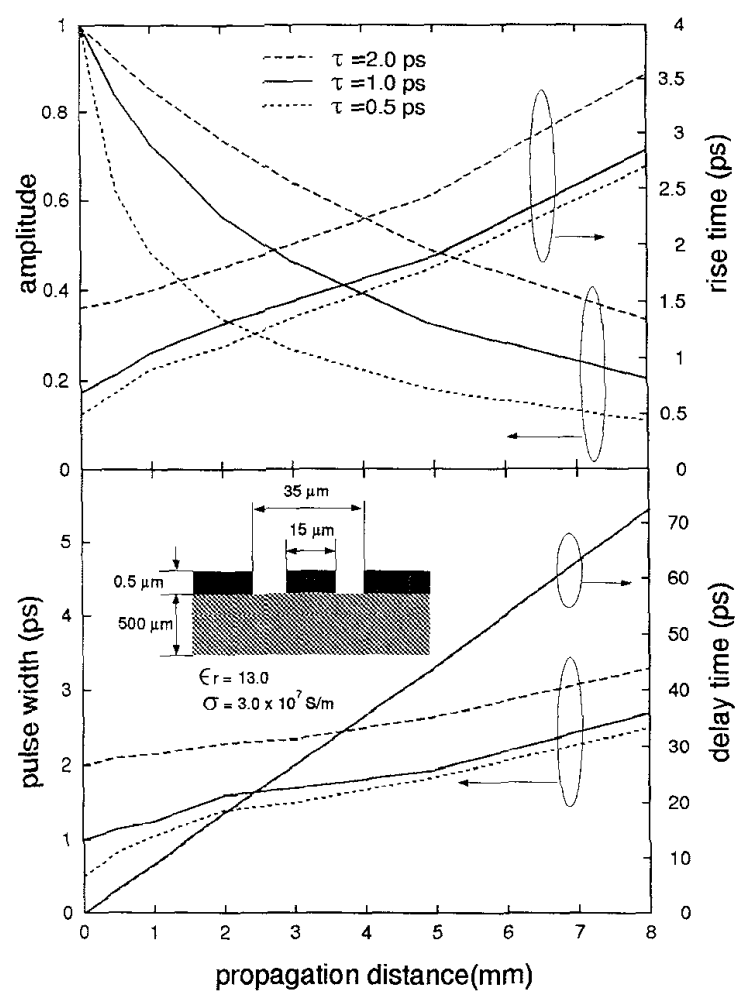

Fig. 6: Peak amplitude, pulse width, rise time, and delay time versus propagation distance with input pulse width $\tau$ as parameters.

[4] J. Y. Ke and C. H. Chen, "Dispersion and attenuation characteristics of coplanar waveguides with finite metallization thickness and conductivity," IEEE Trans. Microwave Theory Tech., vol. 43, pp. 1128-1135, May 1995.

[5] K. C. Gupta, R. Garg, and L. J. Bahl, Microstrip Lines and Slotlines. New York: Artech House, 1979.

[6] S. Gupta, J. F. Whitaker, and G. A. Mourou, "Subpicosecond pulse propagation on coplanar waveguides: experiment and simulation," IEEE Microwave and Guided Wave Lett., vol. 1, pp. 161-163, July 1991. 